\title{
DIHEDRAL ALGEBRAS ARE CYCLIC
}

\author{
LOUIS H. ROWEN ${ }^{1}$ AND DAVID J. SALTMAN ${ }^{2,3}$
}

\begin{abstract}
Any central simple algebra of degree $n$ split by a Galois extension with dihedral Galois group of degree $2 n$ is, in fact, a cyclic algebra. We assume that the centers of these algebras contain a primitive $n$th root of unity.
\end{abstract}

In his book [1], Albert has a proof that every division algebra of degree 3 is cyclic. In this paper we will generalize this result, and derive the theorem below. Our argument is very close to that of Albert, and arose as part of a close examination of his proof. Fix $n$ to be an odd positive integer, and $F$ a field of characteristic prime to $n$. Denote by $D_{n}$ the dihedral group of order $2 n$. We assume the reader is familiar with the basics of the theory of finite dimensional simple algebras as presented, for example, in Albert's book.

TheOREM 1. Let $D$ be a simple algebra of degree $n$ with center $F$. Assume $F$ contains a primitive nth root of one. Suppose $D$ is split by a Galois extension $L / F$ with Galois group $D_{n}$. Then $D$ is a cyclic algebra, that is, $D$ is split by a cyclic Galois extension of degree $n$.

Before beginning the proof of the above theorem, we note that Snider [4] has already shown that such $D$ are similar (in the Brauer group) to a tensor product of cyclic algebras.

The group $D_{n}$ is generated by $\sigma, \tau$ where $\sigma^{n}=1, \tau^{2}=1$ and $\sigma \tau=\tau \sigma^{-1}$. Given $L / F$ as in the theorem, we let $K$ denote the fixed field of $\tau$ in $L$, and $L_{0}$ the fixed field of $\sigma$. Clearly $L$ splits $D \otimes_{F} K$, which also has degree $n$. Since $L / K$ has degree 2 and $n$ is odd, $D \otimes_{F} K$ is already split. That is, $K$ splits $D$. So $K$ can be assumed to be a subfield of $D$.

Since $L / L_{0}$ is cyclic, there is an $\alpha \in L$ such that $\alpha^{n} \in L_{0}$ and $\sigma(\alpha)=\rho \alpha$ where $\rho$ is a primitive $n$th root of one. View $L$ as a subfield of $D \otimes_{F} L_{0}$. Then there is a unit $\beta \in D \otimes_{F} L_{0}$ such that $\alpha \beta=\rho \beta \alpha$. Let $\tau$ act on $D \otimes_{F} L_{0}$ via its action on $L_{0}$. This next lemma, essentially in [1, p. 177], is included here because it is not stated there with the generality we require. For convenience, we provide a proof.

LEMMA 2. We may assume $\tau(\beta)=\beta^{-1}$.

Received by the editors February 17, 1981.

1980 Mathematics Subject Classification. Primary 16A39, 12 E15.

Key words and phrases. Central simple algebra, cyclic algebra.

${ }^{1}$ The first author is currently on sabbatical at Yale University.

${ }^{2}$ The second author is grateful for support under a N.S.F. Postdoctoral Fellowship and a Sloan Foundation Fellowship for Basic Research.

${ }^{3}$ Both authors would like to thank the Yale Health Plan for its hospitality. 
Proof. Since $\alpha(\tau(\alpha))=\tau \sigma^{-1}(\alpha)=\rho^{-1} \tau(\alpha)$, we have $\tau(\alpha)=a \alpha^{-1}$, where $a \in L_{0}$. In fact, since $\alpha=\tau^{2}(\alpha)=\tau\left(a \alpha^{-1}\right)=\tau(a) a^{-1} \alpha$, we have $\tau(a)=a$ and so $a \in F$.

Let $r=(n+1) / 2$ and set $\beta^{\prime}=\beta^{r} \tau(\beta)^{-r}$. Compute that $\alpha \beta^{\prime}=\rho \beta^{\prime} \alpha$ and $\tau\left(\beta^{\prime}\right)$ $=\beta^{\prime-1}$. Q.E.D.

With $\beta$ as in Lemma $2, L_{0}(\beta)$ is Galois over $F$ with group $D_{2 n}$. (If $D$ is not a division algebra, $L_{0}(\beta)$ may be a direct sum of fields, but this does not affect our argument.) Applying Lemma 2 again (reversing the roles of $\alpha$ and $\beta$ ), we may also assume $\tau(\alpha)=\alpha^{-1}$. To prove the theorem, it suffices to find $\eta \in D$ such that $0 \neq \eta^{n} \in F$ and $\eta^{m} \notin F$ for $1 \leqslant m<n$. That $\eta \in D$ is equivalent to saying $\eta \in D \otimes_{F} L_{0}$ and $\eta$ is fixed by $1 \otimes \tau$. The key step in finding such an $\eta$ is the following.

Lemma 3. Suppose $c \in K$. Set $\eta=\left(\beta+\beta^{-1}\right) c$. Denote by $X^{n}+c_{1} X^{n-1}$ $+\cdots+c_{n}$ the characteristic polynomial of $\eta$. Then $c_{i}=0$ for all $i$ odd such that $1 \leqslant i<n$.

Proof. To start off with, assume $F$ has characteristic 0 . If $r$ is odd and $1<r<n$, then $\eta^{r}$ is a sum of terms of the form $d \beta^{s}$ where $d \in L, s$ is odd, and $-r \leqslant s \leqslant r$. Thus $\eta^{r}$ has reduced trace zero. Using Newton's identity (e.g. [3, p. 135]), this case of the lemma is done.

To prove the lemma in general, we use a specialization argument, which we only outline. Let $R_{1}$ be the number ring $Z(\rho)(1 / n)$. Set $T$ to be the localized polynomial ring $R_{1}\left[x, y, z_{1}, \ldots, z_{n}\right](1 / w)$ where $w$ is the $\sigma$ norm of $y x\left(x^{2}-1\right)\left(y^{2}-1\right)$. Let $D_{n}$ act on $T$ via $\sigma(x)=\rho x, \tau(x)=x^{-1}, \sigma(y)=y, \tau(y)=y^{-1}, \tau\left(z_{i}\right)=z_{i}$, and $\sigma\left(z_{i}\right)=$ $z_{i+1}$ (indices modulo $n$ ). The fixed ring of $D_{n}$ on $T$ we call $R$, while we let $S$ denote the fixed ring of $\sigma$ on $T$. One can show that $T / R$ is a Galois extension of commutative rings with group $D_{n} . T / R$ is a generic model for $L / F$, with $S$ corresponding to $L_{0}, x$ corresponding to $\alpha, y$ corresponding to $\beta^{n}$, and $z_{1}$ corresponding to $c$.

Form the cyclic Azumaya algebra $A=(T / S, \sigma, y)$, and take $v \in A$ such that $v^{n}=y$ and $v^{-1} a v=\sigma(a)$ for $a \in T$. Extend $\tau$ to $A$ by setting $\tau(v)=v^{-1}$. Of course, $A$ is a generic model for $D \otimes_{F} L_{0}$, with $v$ corresponding to $\beta$.

Consider $\eta^{\prime}=\left(v+v^{-1}\right) z_{1}$. Let $\eta^{\prime}$ have characteristic polynomial $X^{n}+d_{1} X^{n-1}$ $+\cdots+d_{n}$, where $d_{i} \in R$. By considering $A \otimes_{Z} Q$, we conclude that $d_{i}=0$ if $i$ is odd and less than $n$. Then lemma now follows by specialization. Q.E.D.

To finish the proof of Theorem 1 , set $\eta=\left(\beta+\beta^{-1}\right)\left(\alpha+\alpha^{-1}\right)^{-1}$, and suppose $X^{n}+c_{1} X^{n-1}+\cdots+c_{n}$ is the characteristic polynomial of $\eta$. We have $c_{1}=c_{3}$ $=\cdots=c_{n-2}=0$. We claim $\beta+\beta^{-1}$, and hence $\eta$, can be assumed to be a unit. But $\beta+\beta^{-1}$ has reduced norm $\beta^{n}+\beta^{-n} \in F$ so it suffices to show that we can assume $\beta^{n}+\beta^{-n} \neq 0$. But if $\beta^{n}+\beta^{-n}=0$ then $\left(\beta^{n}\right)^{2}=1$ so $\beta^{n}=-1$ and $D$ is a split algebra, a case which is trivial. Now $\eta^{-1}=\left(\alpha+\alpha^{-1}\right)\left(\beta+\beta^{-1}\right)^{-1}$ has characteristic polynomial $X^{n}+\left(c_{n-1} / c_{n}\right) X^{n-1}+\cdots+\left(1 / c_{n}\right)$. Lemma 3 also applies to $\eta^{-1}$ by symmetry, $c_{n-1}=c_{n-3}=\cdots=c_{2}=0$. Thus $\eta^{n}=-c_{n} \in F$. It is trivial to see that $\eta^{m} \notin F$ for $m<n$, and so the theorem is proved. 
As a final remark, note that the result corresponding to Theorem 1 for $D_{p}$ and fields of characteristic $p$ is a consequence of the more general theorem in [2].

\section{REFERENCES}

1. A. A. Albert, Structure of algebras, Amer. Math. Soc. Colloq. Publ., vol. 24, Amer. Math. Soc., Providence, R.I., 1939.

2. A note on normal division algebras of prime degree, Bull. Amer. Math. Soc. 44 (1938), 649-652.

3. N. Jacobson, Basic algebra. I, Freeman, San Francisco, Calif., 1974.

4. R. Snider, Is the Brauer Group generated by cyclics?, Ring Theory (Waterloo, 1978), (D. Handelman and J. Lawrence, Eds.), Lecture Notes in Math., vol. 734, Springer-Verlag, Berlin and New York, 1979.

Department of Mathematics, Bar Ilan University, Ramat Gan, Israel

Department of Mathematics, Yale University, New Haven, ConNecticut 06520 